\title{
Photonic RF fractional Hilbert transformers and spectral filters based on 49GHz FSR soliton crystal microcombs
}

\author{
Xingyuan Xu, ${ }^{1}$ Mengxi Tan, ${ }^{1}$ Jiayang Wu, ${ }^{1}$ Thach G. Nguyen, ${ }^{2}$ Sai T. Chu, ${ }^{3}$ Brent E. Little, ${ }^{4}$ Roberto \\ Morandotti, ${ }^{5,6}$ Arnan Mitchell, ${ }^{2}$ and David J. Moss ${ }^{1 *}$ \\ ${ }^{1}$ Centre for Micro-Photonics, Swinburne University of Technology, Hawthorn, VIC 3122, Australia. \\ ${ }^{2}$ ARC Centre of Excellence for Ultrahigh-bandwidth Devices for Optical Systems (CUDOS), RMIT University, Melbourne, VIC 3001, Australia. \\ ${ }^{3}$ Department of Physics, City University of Hong Kong, Tat Chee Avenue, Hong Kong, China. \\ ${ }^{4}$ State Key Laboratory of Transient Optics and Photonics, Chinese Academy of Science, Xi'an, China. \\ ${ }^{5}$ INRS-Énergie, Matériaux et Télécommunications, 1650 Boulevard Lionel-Boulet, Varennes, Québec, J3X 1S2, Canada. \\ ${ }^{6}$ Institute of Fundamental and Frontier Sciences, University of Electronic Science and Technology of China, Chengdu 610054, China.
} Abstract: We report photonic RF fractional Hilbert transformers and filters based on a $49 \mathrm{GHz}$ soliton crystal micro-comb source. By employing up to 80 wavelengths and controlling the channel weights, diverse transfer functions are achieved.

\section{Introduction}

Photonic RF filters have attracted great interest during the past two decades, due to advantages including large operation bandwidths, highly reconfigurable filter shapes, as well as rapid tunability and strong immunity to electromagnetic interference [1-2]. A wide range of photonic RF filters have been proposed using either coherent or incoherent structures, [2] and made decent advances such as achieving narrow bandwidths down to the $\mathrm{MHz}$ level. The micro-comb sources [3], particularly those based on CMOS-compatible platforms [3], offer distinct advantages over traditional multi-wavelength sources, such as the potential to provide a much higher number of wavelengths as well as greatly reduced footprint and complexity [4-8]. To miniaturize photonic RF filters and enhance the performance, we employ an integrated microcomb source to establish parallel wavelength channels for the transversal and channelized filters. A coherent soliton crystal microcomb was employed, which offers a low free spectral range of $49 \mathrm{GHz}$ that supports up to 80 channels in the optical $\mathrm{C}$ band.

Here, we demonstrate photonic RF fractional signal processors including Hilbert transformers (HT) [8], and RF filters using 80-tap transversal structures [9] and channelized structures that have $117 \mathrm{MHz}$ resolution [10]. By arbitrarily controlling the weights of the wavelength channels, diverse transfer functions were achieved, including fractional HTs with tunable fractional orders, lowpass, Gaussian-apodized filters, and bandpass filters with tunable centre frequencies and bandwidths. For the fractional signal processor, orders ranging from 0.166 to 1 are achieved with an operation bandwidth of $16.45 \mathrm{GHz}$. For the transversal filter, a 4-time $\mathrm{Q}$ factor enhancement was achieved (in contrast to previously demonstrated 20-tap microcomb based filter [5]), together with tunable centre frequencies and an out-of-band rejection ratio up to over $48 \mathrm{~dB}$. The channelized filter shows a resolution of $117 \mathrm{MHz}$, together with reconfigurable filtering shapes. The experimental results match well with theoretical calculations, verifying the feasibility of our approach.

\section{Theory and experimental results}

To generate Kerr micro-combs, the pump power was amplified to $\sim 30.5 \mathrm{dBm}$ and the wavelength was swept across a resonance of the active MRR. As the detuning was changed, distinctive 'fingerprint' optical spectra of soliton crystals were observed. The generated comb was spectrally shaped by two stages of Waveshapers (Finisar, 4000S) to precisely achieve desired channel weights. Then the input RF signal was multi-cast onto each comb line via an electro-optical modulator. To achieve transversal structures (Fig. 1), the RF input was broadcast via an intensity modulator and progressively delayed by a spool of single-mode fibre to achieve the required taps. As a result, a high spectral resolution was achieved for both the filter and the HT, together with a significantly improved tunable centre frequency covering the RF spectra range and a high out-of-band rejection of $48.9 \mathrm{~dB}$ (Fig. 2(g, h)) for the former, and a reconfigurable fractional order from 0.166 to 1 for the latter (Fig. 2(a-f)). To achieve channelized filters, the RF input was broadcast via a phase modulator and fed to the passive MRR for spectral slicing. The through-port transmission of the passive MRR was employed to perform phase-to-intensity modulation conversion by filtering out the lower sideband and, at the same time, serves as a high-Q Vernier comb filter for high-resolution RF bandwidth scaling. As shown in Fig. 2(i, j), programmable arbitrary transfer functions for RF spectral shaping with an operation bandwidth of $4.64 \mathrm{GHz}$, together with a high resolution of $117 \mathrm{MHz}$ were achieved. Finally, we note that more compact dispersive devices can be achieved, even offering tunability, with many approaches including etalon tunable dispersion compensators [11-15]. 

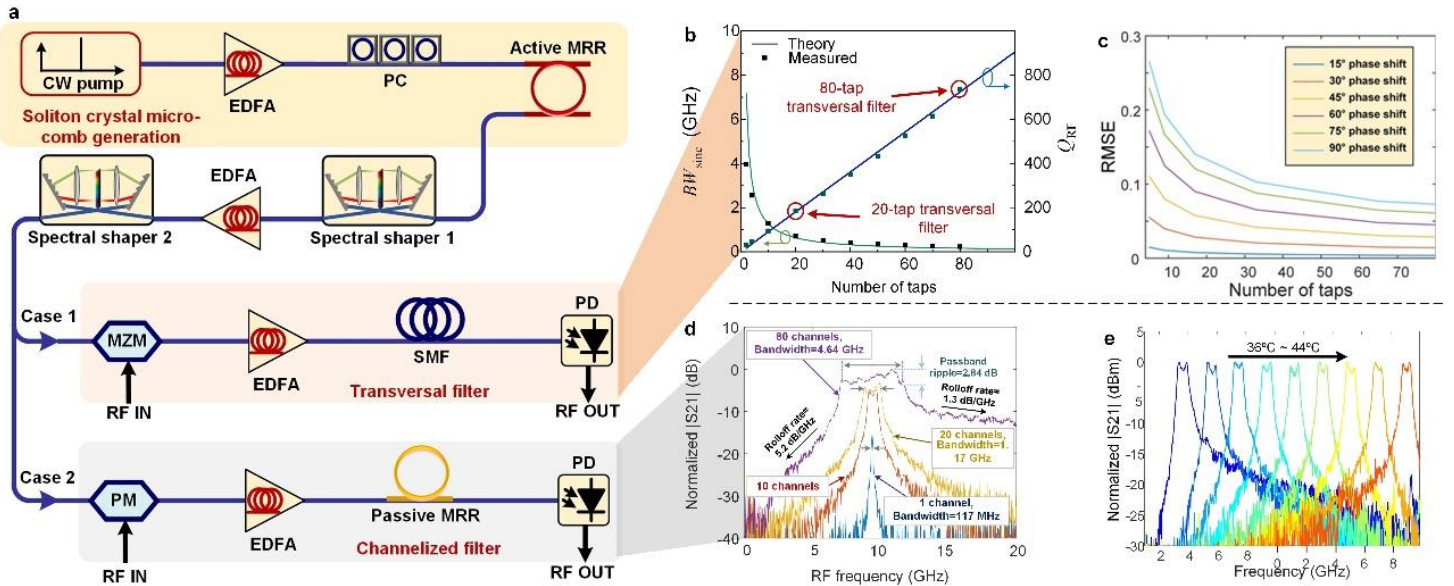

Fig. 1. (a) Schematic diagram of the photonic RF transversal filter based on an integrated 49GHz-spacing micro-comb source. EDFA: erbiumdoped fiber amplifier. PC: polarization controller. MRR: micro-ring resonator. MZM: Mach-Zehnder modulator. SMF: single mode fiber. PD: photodetector. PM: phase modulator. Demonstrated performance enhancement of (b) the transversal filter's Q factor, (c) the discrepancies in the HT's amplitude responses, (d, e) operation bandwidth of the channelized filters.
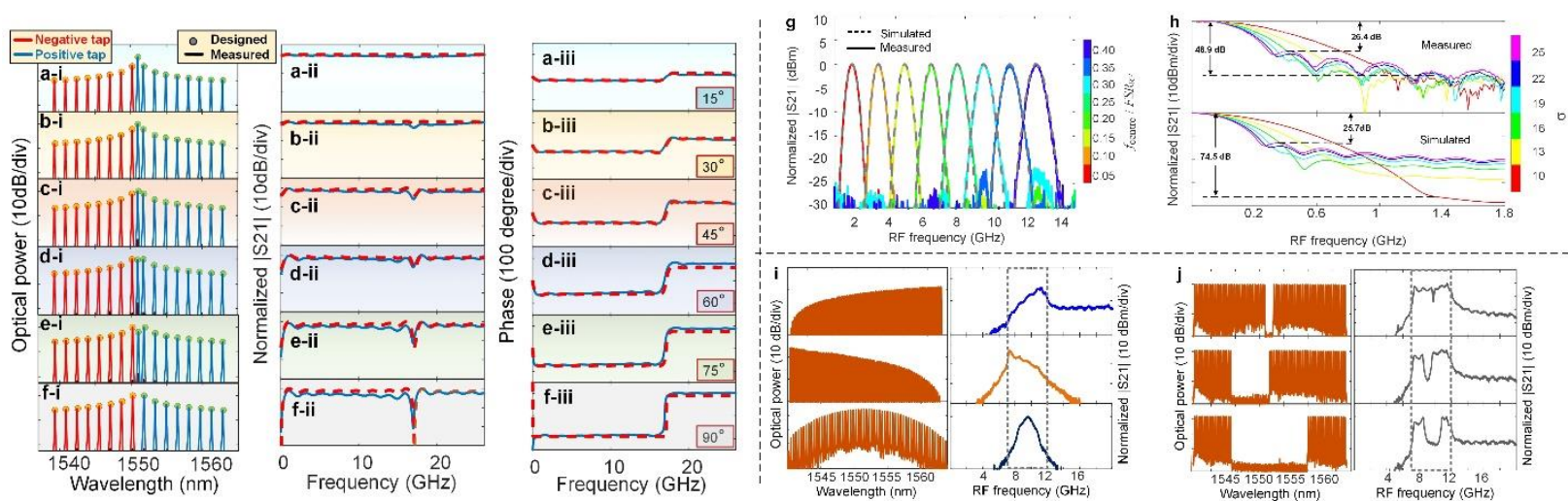

Fig. 2. Demonstration of (a-f) tunability of the HT's fractional order, (g) centre-frequency tunability and (h) Gaussian apodization of the transversal filter, and (i) reconfigurable filtering shapes and (j) binary passbands of the channelized filter.

\section{Conclusions}

We have demonstrated photonic RF signal processors based on soliton crystal microcombs. This approach offers a greatly reduced footprint, lower complexity, and potentially lower cost, representing a solid step towards the miniaturization of high performance photonic RF systems [16-25].

\section{References}

[1] J. Capmany, and D. Novak, "Microwave photonics combines two worlds," Nat. Photonics, vol. 1, no. 6, pp. 319-330, Jun. 2007.

[2] J. P. Yao, "Microwave Photonics," J. Lightwave Technol., vol. 27, no. 1-4, pp. 314-335, Jan-Feb. 2009.

[3] D. J. Moss, R. Morandotti, A. L. Gaeta, and M. Lipson, "New CMOS-compatible platforms based on silicon nitride and Hydex for nonlinear optics," Nat. Photonics 7, 597-607 (2013).

[4] X. Xu et al., "Photonic microwave true time delays for phased array antennas using a $49 \mathrm{GHz}$ FSR integrated optical micro-comb source [Invited]," Photonics Res, vol. 6, no. 5, pp. B30-B36, May. 2018.

[5] X. Xu et al., "Advanced RF and microwave functions based on an integrated optical frequency comb source," Opt. Express, vol. 26, no. 3, pp. 2569-2583, Feb. 2018.

[6] X. Xu et al., "Reconfigurable broadband microwave photonic intensity differentiator based on an integrated optical frequency comb source," APL Photonics, vol. 2, no. 9, 096104. 2017.

[7] X. Xu et al., "Broadband RF Channelizer based on an Integrated Optical Frequency Kerr Comb Source," Journal of Lightwave Technology, vol. 36, no. 19, pp.4519-4526. 2018.

[8] M. Tan et al., "Microwave and RF photonic fractional Hilbert transformer based on a 50GHz Kerr micro-comb," Journal of Lightwave Technology, vol. 37, no. 24, pp. 6097 - 6104. 2019. DOI: 10.1109/JLT.2019.2946606

[9] X. Xu et al., "Advanced Adaptive Photonic RF Filters with 80 Taps Based on an Integrated Optical Micro-Comb Source," Journal of Lightwave Technology, vol. 37, no. 4, pp. 1288-1295. 2019.

[10] X. Xu et al., "High performance RF filters via bandwidth scaling with Kerr micro-combs," APL Photonics, vol. 4, no. 2, pp. 026102.2019.

[11] D.J. Moss, M.Lamont, S.Mclaughlin, G.Randall, P.Colbourne, S.Kiran and C.A.Hulse,'Tunable dispersion and dispersion slope compensators for 10Gb/s using all-pass multicavity etalons", IEEE Photonics Technology Letters, vol. 15, no. (5), 730-732 (2003). DOI: 10.1109/LPT.2003.809921. 
[12] L.M. Lunardi, D.Moss, S.Chandrasekhar, L.L.Buhl, A. Hulse, P.Colbourne, G.Randall, S.Mclaughlin, "Tunable dispersion compensators based on multi-cavity all-pass etalons for 40Gb/s systems", Journal of Lightwave Technology, vol. 20, no. 12, 2136 (2002). DOI: $10.1109 /$ JLT.2002.806768.

[13] D.J. Moss, S.Mclaughlin, G.Randall, M.Lamont, M. Ardekani, P.Colbourne, S.Kiran and C.A.Hulse, "Multichannel tunable dispersion compensation using all-pass multicavity etalons", paper TuT2 Optical Fiber Communications Conference, Anaheim (2002). Postconference Technical Digest (IEEE Cat. No.02CH37339). Opt Soc. America. Part vol.1, 2002, pp. 132-3. Washington, DC, USA.

[14] D.J.Moss, et al., "Tunable dispersion compensation at $10 \mathrm{~Gb} / \mathrm{s}$ and $40 \mathrm{~Gb} / \mathrm{s}$ using multicavity all-pass etalons", Optical Fiber Communications Conference (OFC) paper TuD1, page 162, Atlanta, GA, March (2003). Post-conference Digest (IEEE Cat. No.03CH37403). Opt. Soc. America. Part vol.1, 2003, pp. 162-3. Washington, DC, USA.

[15] L.M. Lunardi, D.J. Moss, S. Chandrasekhar, L.L. Buhl, "An Etalon-Based Tunable Dispersion Compensator (TDC) Device for 40-Gbit/s Applications", European Conference on Optical Communications (ECOC), Paper 5.4.6 Copenhagen, Sept. (2002). IEEE. Part vol. 2, 2002, pp. 2 vol. 2. Piscataway, NJ, USA. Print ISBN: 87-90974-63-8. Print ISBN: 87-90974-63-8.

[16] X. Xu, M. Tan, J. Wu, R. Morandotti, A. Mitchell, and D. J. Moss, "Microcomb-based photonic RF signal processing," IEEE Photonics Technology Letters vol. 31, no. 23, pp. 1854-1857. 2019. DOI: 10.1109/LPT.2019.2940497

[17] X. Xu et al, "Photonic RF phase-encoded signal generation with a microcomb source", Journal of Lightwave Technology vol. 38, no.7, pp. 1722-1727. 2020. DOI:10.1109/JLT.2019.2958564.

[18] X.Xu et al, "Broadband microwave frequency conversion based on an integrated optical micro-comb source", Journal of Lightwave Technology vol. 38, no. 2, pp. 332-338. 2020.

[19] M. Tan et al, "Broadband microwave and RF fractional differentiator using photonics", IEEE Transactions on Circuits and Systems: Express Briefs, Early Access (2020). DOI:10.1109/TCSII.2020.2965158.

[20] X. Xu et al, "Photonic RF and microwave integrator with soliton crystal microcombs", IEEE Transactions on Circuits and Systems: Express Briefs, Early Access (2020). DOI:10.1109/TCSII.2020.2995682

[21] X. Xu et al, "Broadband photonic radio frequency channelizer with 90 channels based on a soliton crystal microcomb", Journal of Lightwave Technology Early Access, vol. 38. 2020. doi: 10.1109/JLT.2020.2997699

[22] M. Tan et al, "Photonic RF arbitrary waveform generator based on a soliton crystal micro-comb source", Journal of Lightwave Technology Early Access vol. 38. 2020. DOI:10.1109/JLT.2020.3009655.

[23] X. Xu et al, "Photonic perceptron based on a Kerr microcomb for scalable high speed optical neural networks", Laser and Photonics Reviews, vol.14. 2020. DOI:10.1002/lpor.202000070.

[24] M. Tan et al, "Photonic RF and microwave filters based on $49 \mathrm{GHz}$ and $200 \mathrm{GHz}$ Kerr microcombs", Optics Communications, vol. 465, Article: 125563.2020 . DOI:10.1016/j.optcom.2020.125563.

[25] J. Wu et al, "RF photonics: An optical micro-combs' perspective", IEEE Journal of Selected Topics in Quantum Electronics vol. 24, no. 4, pp. 1-20, Article Number: 6101020. 2018. DOI: 10.1109/JSTQE.2018.2805814. 